\title{
Transmission Electron Microscopic Confirmation of the Morphological Predictions of the Two-Site Model for Hydroxyapatite Dissolution ${ }^{1}$
}

\author{
EARL N. GRIFFITH, ASHOK KATDARE, JEFFREY L. FOX, ${ }^{2}$ \\ AND WILLIAM I. HIGUCHI \\ College of Pharmacy, The University of Michigan, Ann Arbor, Michigan 48109
}

Received October 7, 1977; accepted May 30, 1978

\begin{abstract}
Hydroxyapatite (HAP) crystals were dissolved under two types of solution conditions designed to produce contrasting dissolution morphologies according to the recently proposed two-site model for HAP dissolution [Fox, J. L., Higuchi, W. I., Fawzi, M. B., and Wu, M. S., J. Colloid Interface Sci., 67, 312 (1978)]. In accordance with this model, dissolution into partially saturated buffers proceeded via formation of holes in the crystals, while dissolution of comparable amounts of material into completely unsaturated buffers showed no such holes. These results show that the two dissolution sites inferred from dissolution kinetics experiments are, in fact, physically distinct sites and that dissolution from site No. 1 results in hole formation. The physical location of site No. 2 is not yet certain.
\end{abstract}

Recently, a model involving two distinct types of dissolution sites has been proposed to explain the dissolution behavior of hydroxyapatite. This model was based largely on the results of measurements of the dissolution kinetics of hydroxyapatite for a large number of experimental conditions. Although these experimental results were consistent with the proposed model, they were unable to unambiguously show that hydroxyapatite did indeed possess two distinct dissolution sites as opposed to one site with fairly complex kinetics. Other types of experimental observations such as morphological studies and remineralization studies were consistent with the model, but none provided a direct test of the model's validity. The purpose of this paper is to describe the first direct test of the two-site model.

\footnotetext{
${ }^{1}$ This investigation supported by NIDR grant DE01830.

${ }^{2}$ To whom correspondence should be addressed.
}

\section{BASIS FOR THE TEST}

The primary hypothesis of the two-site model is that the dissolution kinetics of hydroxyapatite are governed by two distinct types of dissolution sites, each with its own apparent solubility. These apparent solubilities are significantly different from each other, and significantly less than the equilibrium solubility for hydroxyapatite. The model proposes that a given site will dissolve only if the solution immediately adjacent to the crystal is undersaturated with respect to that site. On the basis of our kinetic experiments we have concluded that the less soluble of the two sites (site No. 2) has a much larger apparent kinetic rate constant and therefore dominates dissolution in completely unsaturated solutions. The more soluble site (site No. 1) has a lower apparent rate constant and is therefore important only when the adjacent solution is oversaturated with respect to site No. 2. 


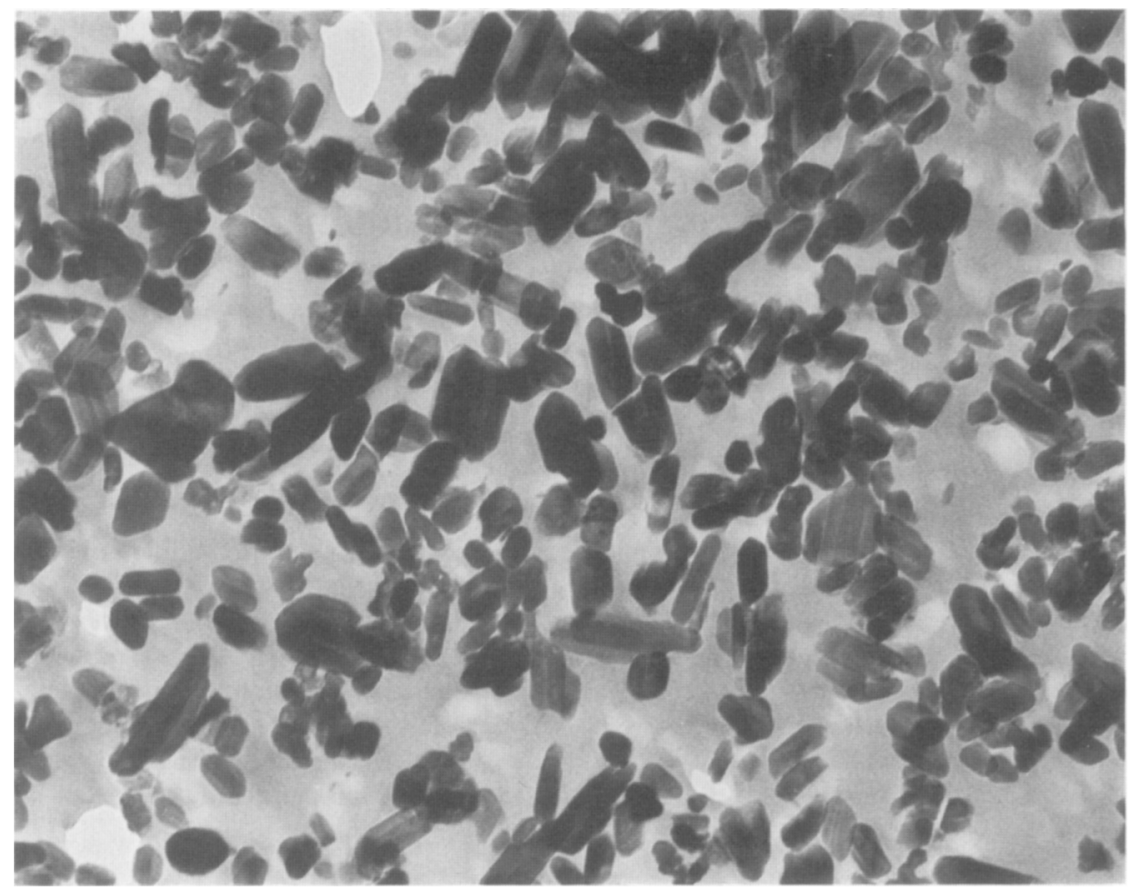

Fig. 1. Untreated hydroxyapatite crystals. Width of field is $20,000 \AA$.

Based on morphological studies done by others, we have tentatively postulated that dissolution from site No. 1 might correspond to the formation of holes along the long axis of the crystal whereas site No. 2 dissolution might correspond to dissolution over the entire basal plane surface, leading to a shortening of the crystal. It is this hypothesis that we intend to test.

\section{METHODS AND MATERIALS}

Synthetic hydroxyapatite, prepared by precipitation and digestion near $100^{\circ} \mathrm{C}$, was provided by Dr. P. R. Patel of the National Bureau of Standards.

A $25-\mathrm{mg}$ sample of the hydroxyapatite crystals was treated in one of two ways. In the first method the sample was placed in a glass beaker and 50,100 , or $200 \mathrm{ml}$ of a solution partially saturated with respect to hydroxyapatite $(3.5 \mathrm{mM}$ in total calcium and in total phosphate in a pH 4.5 acetate buffer with ionic strength adjusted to 0.5 by addition of $\mathrm{NaCl}$ ) was added. The sample was maintained at $30^{\circ} \mathrm{C}$ and stirred at high speed with a magnetic stirrer for 20,40 , or $80 \mathrm{~min}$ ( $25 \mathrm{mg}$ of HAP/50 ml for $20 \mathrm{~min}, 25$ $\mathrm{mg}$ of $\mathrm{HAP} / 100 \mathrm{ml}$ for $40 \mathrm{~min}$, and $25 \mathrm{mg}$ of $\mathrm{HAP} / 200 \mathrm{ml}$ for $80 \mathrm{~min})$. The solutions were then vacuum filtered through $0.22-\mu \mathrm{m}$ Millipore filters and the filtrate was analyzed for calcium. The crystals trapped on the Millipore filters were rinsed with doubledistilled water and dried overnight in an oven at $60^{\circ} \mathrm{C}$ and then embedded in Epon 812 formula $\mathrm{B}$ for sectioning.

In the second method the sample of HAP crystals is placed between two 4.25$\mathrm{cm}$ Whatman No. 1 qualitative filter papers and placed in a Millipore filter funnel. Volumes of 200,600 , or $1000 \mathrm{ml}$ of $\mathrm{pH} 4.5$ acetate buffer, ionic strength 0.5 , are pulled by vacuum through the filter paper crystal sandwich at a rate in the range of $140-350$ $\mathrm{ml} / \mathrm{min}$. After the solution has passed through, the filtrate is analyzed for calcium. The crystals trapped in the sandwich are rinsed with double-distilled water and dried overnight at $60^{\circ} \mathrm{C}$. The crystals are then em- 


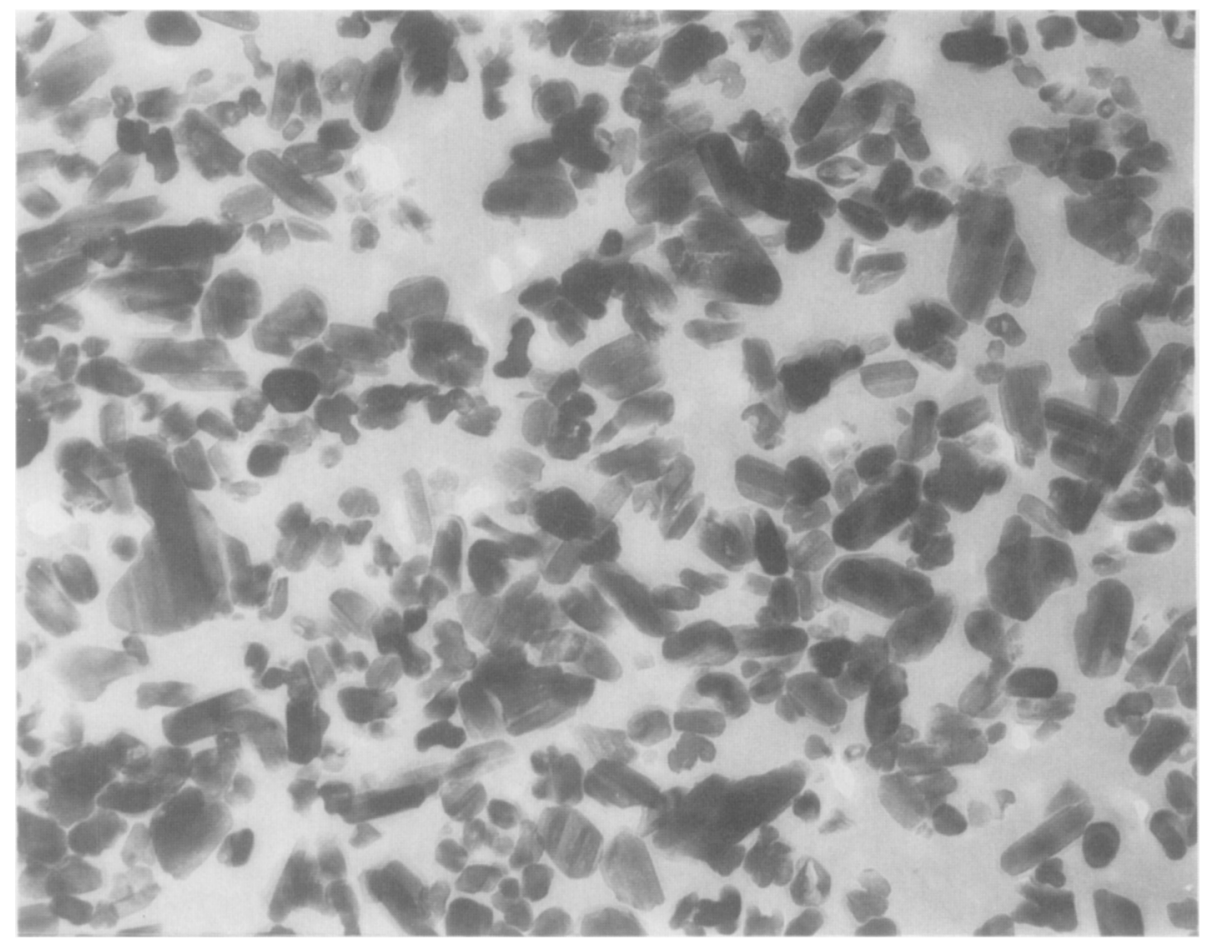

FIG. 2. Hydroxyapatite crystals exposed to completely unsaturated acetate buffer for $5 \mathrm{~min}$, resulting in dissolution of $56 \%$ of original sample. Width of field is $20,000 \AA$.

bedded in Epon 812 formula $B$ and sectioned with a diamond knife in a Huxley ultramicrotome at a speed of $1.8 \mathrm{~mm} / \mathrm{sec}$, giving a section with an area of no more than $0.25 \mathrm{~mm}^{2}$ and a thickness of 250 or $500 \AA$.

The finished sections from either procedure are picked up on 200 mesh screens and examined and photographed on a Siemens Elmiskop 1A at a magnification of $10,000 \times$ on the $2 \times 3$ negative.

\section{RESULTS}

Typical transmission electron micrographs of untreated crystals, and those subjected to the two treatments described above are shown in Figs. 1-3. Figure 1 shows the untreated control sample. These crystals are intact and show virtually no evidence of hole formation. These are in great contrast with Fig. 3, the crystals subjected to dissolution under partial saturation conditions. The mineral loss from this sample was $37 \%$ of the total starting ma- terial. As the micrograph clearly shows, hole formation was a major mechanism of demineralization for this sample. Figure 2 represents a sample $56 \%$ dissolved under sink conditions. There are no holes of a magnitude comparable to those in Fig. 3, but close examination reveals tiny "pinpricks" on some of the crystals which probably represent the beginning of hole formation. These have not progressed as far as the holes in Fig. 3, partly because the sink condition experiment was for a much shorter time (5 min as compared to $40 \mathrm{~min}$ for the partial saturation treatment resulting in the material shown in Fig. 3).

\section{DISCUSSION}

These results, the two-site model for dissolution kinetics and the morphological observations of Scott et al. (1) Swancar et al. (2), Arends et al. (3), and Arends (4), combine to give a comprehensive picture 


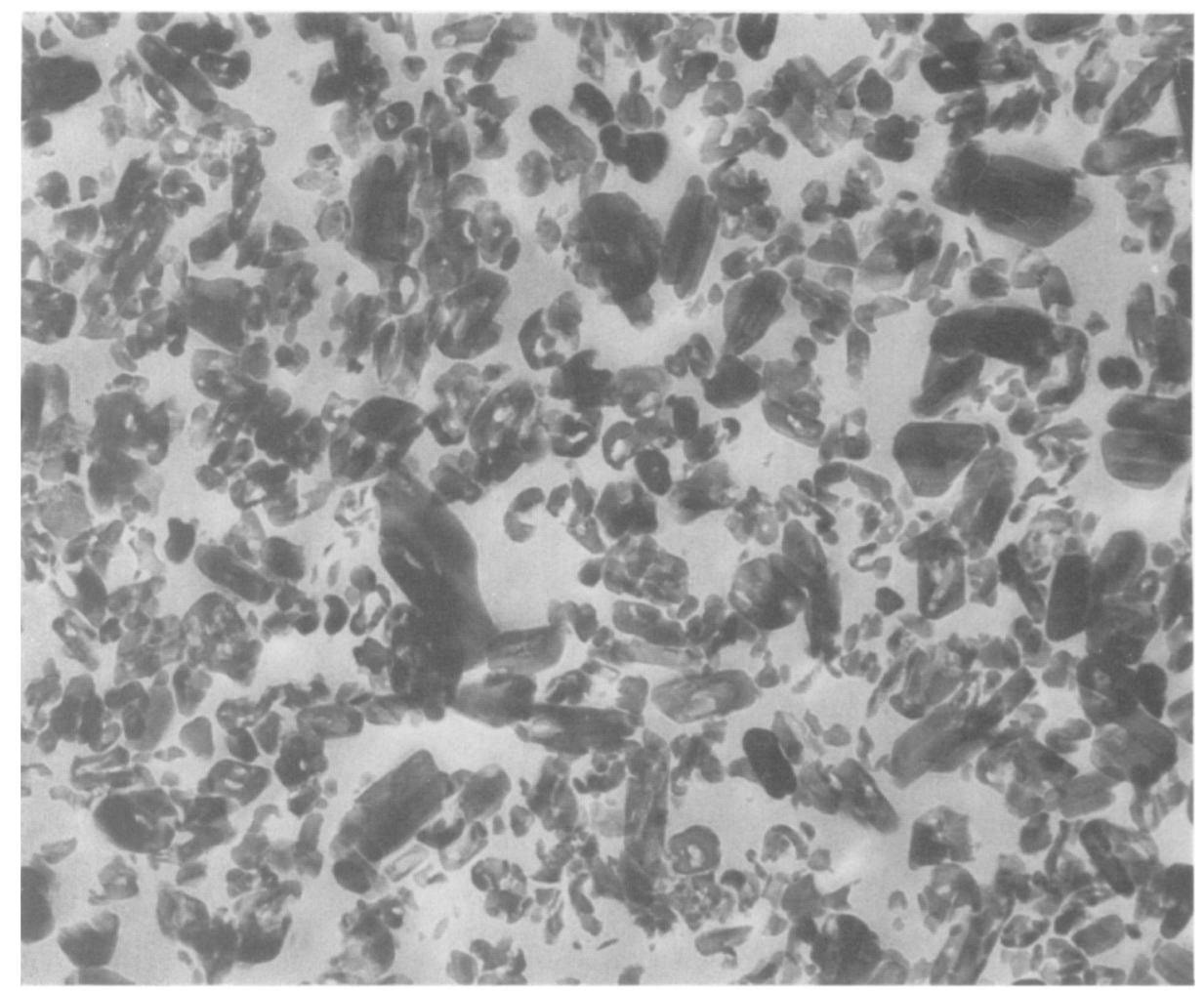

FIG. 3. Hydroxyapatite crystals exposed to partially saturated acetate buffer for 40 min, resulting in dissolution of $37 \%$ of original sample. Width of field is $20,000 \AA$.

of the dissolution behavior of synthetic hydroxyapatite and, by inference, human dental enamel.

Behavior at the single crystal level is explainable in terms of a model with two types of dissolution sites, each of which can contribute to dissolution only when the immediately surrounding solution is undersaturated with respect to the apparent solubility of the site. The more soluble of these two sites, which we have called site No. 1, is responsible for the dissolution behavior in partially saturated solutions which are supersaturated with respect to the less soluble site No. 2. Measurements of hydroxyapatite dissolution kinetics indicate that site No. 1 has a smaller apparent dissolution rate constant than site No. 2, but this may reflect a smaller population of these sites rather than an actual smaller rate constant. Physically, site No. 1 dissolution results in the hole formation shown in Fig. 3. If this dissolution pattern is the result of ion disengagement along a screw dislocation parallel to the long axis of the crystal as has been suggested (4), then it is reasonable that this process might occur quite readily, as is indicated by the higher apparent solubility of this site. If such sites occupy a small fraction of the crystal surface, as is suggested by their appearance as tiny pinhole-like pits in their early stage of development (Fig. 2), then the low apparent rate constant is to be expected, at least for the initial dissolution rates which were used in developing the two-site model.

Site No. 2, the site with the lower apparent solubility, is the dominant one when dissolution occurs under sink conditions. The physical location of this site has yet to be definitely determined. Comparison of 
the control (Fig. 1) and the sample 56\% dissolved under sink conditions yields no obvious answer as to the location of this site. Because site No. 2 has a high apparent rate constant (compared to site No. 1), it is probable that this site occupies a much larger fraction of the crystal surface, perhaps even an entire face of the crystal.

\section{SUMMARY}

Hydroxyapatite crystals were dissolved under two types of solution conditions designed to produce contrasting dissolution morphologies according to the recently proposed two-site model for dissolution (5). In accordance with this model, dissolution into partially saturated buffers proceeded via formation of holes in the crystals, while dissolution into completely unsaturated buffers showed only tiny "pinpricks" rather than fully developed holes. These results show that the two dissolution sites inferred from dissolution kinetics experiments are, in fact, two physically distinct sites, and that site No. 1 dissolution results in hole formation. The physical location of site No. 2 is not yet certain.

\section{REFERENCES}

1. Scott, D. B., Simmelink, J. W., and Nygaard, V., in "Chemistry and Physiology of Enamel," pp. 6-24. The University of Michigan Press, Ann Arbor, Michigan, 1971.

2. Swancar, J. R., Scott, D. B., Simmelink, J. W., and Smith, T. J., in "Tooth Enamel II" (R. W. Fearnhead and M. V. Stack, Eds.), pp. 233-239. John Wright, Bristol, England, 1971.

3. Arends, J., Berg, v.d. P. J., and Jongeblood, W. L., in "Physicochemimie at Cristallographie des Apatites d'interet Biologique," pp. 389-395. Proceedings of Colloques Internationaux C.W.R.S., 1975.

4. Arends, J., Caries Res. 7, 261 (1973).

5. Fox, J. L., Higuchi, W. I., Fawzi, M. B., and Wu, M. S., J. Colloid Interface Sci, 67, 312 (1978). 